\title{
In situ comparison of A-mode ultrasound tracking system and skin- mounted markers for measuring kinematics of the lower extremity
}

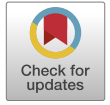

\author{
Kenan Niu ${ }^{a, *}$, Thomas Anijs ${ }^{b}$, Victor Sluiter ${ }^{a}$, Jasper Homminga ${ }^{a}$, André Sprengers ${ }^{b}$, Marco A. Marra ${ }^{b}$, \\ Nico Verdonschot ${ }^{\mathrm{b}}$ \\ ${ }^{a}$ Laboratory of Biomechanical Engineering, Faculty of Engineering Technology, MIRA Institute, University of Twente, Drienerlolaan 5, Enschede, The Netherlands \\ ${ }^{b}$ Orthopaedic Research Lab, Radboud University Medical Center, Radboud Institute for Health Sciences, Nijmegen, The Netherlands
}

\section{A R T I C L E I N F O}

\section{Article history:}

Accepted 3 March 2018

\section{Keywords:}

Knee kinematics

Soft tissue artifacts

Ultrasound

Skin markers

Motion capture

\begin{abstract}
A B S T R A C T
Skin-mounted marker based motion capture systems are widely used in measuring the movement of human joints. Kinematic measurements associated with skin-mounted markers are subject to soft tissue artifacts (STA), since the markers follow skin movement, thus generating errors when used to represent motions of underlying bone segments. We present a novel ultrasound tracking system that is capable of directly measuring tibial and femoral bone surfaces during dynamic motions, and subsequently measuring six-degree-of-freedom (6-DOF) tibiofemoral kinematics. The aim of this study is to quantitatively compare the accuracy of tibiofemoral kinematics estimated by the ultrasound tracking system and by a conventional skin-mounted marker based motion capture system in a cadaveric experimental scenario. Two typical tibiofemoral joint models (spherical and hinge models) were used to derive relevant kinematic outcomes. Intra-cortical bone pins equipped with optical markers were inserted in the tibial and femoral bones to serve as a reference to provide ground truth kinematics. The ultrasound tracking system resulted in lower kinematic errors than the skin-mounted markers (the ultrasound tracking system: maximum root-mean-square (RMS) error $3.44^{\circ}$ for rotations and $4.88 \mathrm{~mm}$ for translations, skin-mounted markers with the spherical joint model: $6.32^{\circ}$ and $6.26 \mathrm{~mm}$, the hinge model: $6.38^{\circ}$ and $6.52 \mathrm{~mm}$ ). Our proposed ultrasound tracking system has the potential of measuring direct bone kinematics, thereby mitigating the influence and propagation of STA. Consequently, this technique could be considered as an alternative method for measuring 6-DOF tibiofemoral kinematics, which may be adopted in gait analysis and clinical practice.
\end{abstract}

(c) 2018 Elsevier Ltd. All rights reserved.

\section{Introduction}

Accurate measurements of joint kinematics are essential to comprehensively understand the mechanism of joint motion. Most of kinematic data on the lower extremity are obtained from motion capture systems, in which the trajectories of skin-mounted markers are recorded to represent the motion of underlying skeletal structure (Lafortune et al., 1992). However, the estimated threedimensional (3D) position and orientation of bone segments and the related kinematics are subject to soft tissue artifacts (STA) (Cereatti et al., 2017). Since the movement of the skin, muscles, and fat relative to the underlying bone is an inevitable phenomenon under dynamic motion tasks (Dumas and Jacquelin, 2017), the inherent mismatch between skin and bone movement

\footnotetext{
* Corresponding author at: Laboratory of Biomechanical Engineering, University of Twente, P.O. Box 217, 7500 AE Enschede, The Netherlands.

E-mail address: niukenan@gmail.com (K. Niu).
}

is difficult to remove under all circumstances (Cappozzo et al. 1995, 1996). It has been reported that STA can cause measurement errors of up to $30 \mathrm{~mm}$ in the thigh (Akbarshahi et al., 2010). The propagation of STA to knee joint kinematics has been reported to lead to average rotational errors of up to $4.4^{\circ}$ and $13.1^{\circ}$ and average translational errors of up to $13.0 \mathrm{~mm}$ and $16.1 \mathrm{~mm}$ for walking and cutting motions, respectively (Benoit et al., 2006).

Extensive researches have been conducted on quantification, assessment, and compensation of STA for different motor tasks (Andersen et al., 2009; Bonnechère et al., 2015; Cappozzo et al., 1997; Charlton et al., 2004; Duprey et al., 2010; Lu and O'Connor, 1999). Multi-body kinematics optimization (MKO) has been used with the intent to compensate the STA and to limit the propagation of STA to joint kinematics estimation (Andersen et al., 2009; Lu and O'Connor, 1999). The typical mechanical linkages representing the knee joint and embedded in MKO are the hinge joint and the spherical joint, which involve major simplifications with respect to the actual joint and reduce the degrees of freedom (DOF) of the joint 
(Ojeda et al., 2014; Reinbolt et al., 2005). The hinge joint only allows the flexion-extension rotation (Andersen et al., 2009). The spherical joint allows all three rotations but no translation (Clement et al., 2017). Researchers have realized that motion analysis research community should make more efforts in search of more advanced subject-specific joint models or error models, or a new measurement modality in order to improve the accuracy of estimated joint kinematics (Andersen et al., 2010; Cereatti et al., 2006; Richard et al., 2017).

Instead of compensating STA from various perspectives of mathematical models and optimizations, we developed a new method to directly measure spatial information of underlying bones in order to produce an effective and valid representation of skeletal motion and the corresponding joint kinematics. A novel ultrasound (US) tracking system was developed to measure tibiofemoral kinematics dynamically, non-invasively, and without radiation. As A-mode ultrasound transducers are capable of detecting underlying bone surfaces non-invasively through multiple layers of soft tissues under dynamic movement, a combination of multiple A-mode ultrasound transducers and conventional skin-mounted markers provides a new approach of measuring the trajectories of multiple A-mode ultrasound transducers attached on the thigh and shank as well as detecting respective underlying bone surfaces from received ultrasound signals. Subsequently, the trajectories of bone surfaces could be obtained and the relevant kinematics can be quantified. To evaluate our system relative to conventional motion capture systems with skin-mounted markers, cadaveric experiments were conducted.

The aim of the presented work is to compare tibiofemoral kinematics derived from our ultrasound tracking system with those kinematic outcomes derived from skin-mounted markers using two typical joint models (hinge and spherical) in cadaveric experiments. Another goal is to demonstrate the potential for being less affected by STA and achieving high accuracy in estimated tibiofemoral kinematics with our ultrasound tracking system.

\section{Material and methods}

\subsection{Experimental setup}

A full body cadaveric specimen (male, $79 \mathrm{~kg}, 179 \mathrm{~cm}$ ) was obtained from and approved by the Anatomy Department of the Radboud University Medical Center (RUMC), Nijmegen, the Netherlands. There was no history of illness, injury, or treatment affecting the knee or hip functions. Tracked intra-cortical bone pins equipped with optical markers were inserted into the right femur (with two pins) and right tibia (with two pins), which provided ground truth kinematics as a reference. A full leg (from the pelvic to foot) CT scan was made at the Department of Radiology of the RUMC using a TOSHIBA Aquilion ONE (TOSHIBA, Tustin, USA) with a voxel size of $0.755 \mathrm{~mm} \times 0.755 \mathrm{~mm} \times 0.500 \mathrm{~mm}$. Subsequently, the femur and tibia were manually segmented using Mimics 17.0 (Materialise N.V., Leuven, Belgium) to generate geometrical surface models of the bones, which were exported in STL format. The femoral and tibial anatomical reference frames were defined based on bone geometries using a method previously described (Miranda et al., 2010). The upper body of the cadaver was fixated with nylon straps in a supine position on a surgical table, while ensuring that the right leg could be manually displaced without any hindrances to its movement.

\subsection{Ultrasound tracking system}

The ultrasound tracking system comprised six customized 3Dtracked ultrasound holders covering six anatomical areas: greater trochanter of the femur, middle femoral shaft, lateral and medial sides of both femoral epicondyles and tibial condyles, middle tibial shaft, and ankle (Fig. 1). Each ultrasound holder consisted of a variable number of A-mode ultrasound transducers (frequency: 7.5 $\mathrm{MHz}$, Imasonic SAS, Voray/l'Ognon, France) and three optical markers that were tracked by Visualeyez VZ4000v system (PTI Phoenix Technologies Inc., Vancouver, Canada) operating at $60 \mathrm{~Hz}$. The ultrasound holders were designed in SolidWorks (Waltham, Massachusetts, USA) and manufactured using Polyamide powder material in 3D printer (EOS Formiga P110, EOS GmbH, Krailling, Germany) to insure high accuracy on their 3D geometrical structures for maintaining the rigidity and stability. Therefore, the spatial relations between each A-mode ultrasound transducer and each optical marker were known parameters. The origin and pointing direction of each ultrasound transducer were parameterized during design. Hence no further physical calibration is required as this is build-in design. The ultrasound detected point can be digitalized through the known origin and pointing direction of each ultrasound transducers and the related spatial relation between each optical marker, when the depth of bone surface is obtained (Fig. 1). In total, $30 \mathrm{~A}$-mode ultrasound transducers and 18 optical markers were installed on six ultrasound holders. The sample rate of the whole ultrasound tracking system was $20 \mathrm{~Hz}$. After attaching all ultrasound holders on the lower extremity, the received ultrasound signals and trajectories of rigidly attached markers were collected to calculate corresponding points on the surface of the bones. Subsequently, these points were fed to a point-cloud registration algorithm modified from the combination of an iterative closest point algorithm (Besl and McKay, 1992) and a perturbation method (Ma and Ellis, 2003) with weighting factors to estimate the position and orientation of the bone model in 3D space. The weighting factors were associated with the intensity (i.e. 'reliability') of the detected peak of the tissue-bone boundary. Finally, tibiofemoral kinematics were quantified from the estimated bone positions per time-frame of acquisition using the established anatomical reference frames (Grood and Suntay, 1983). The relevant data acquisition and processing have been described previously in detail (Niu et al., 2017). The trajectories of inserted intra-cortical bone pins and attached ultrasound holders as well as received ultrasound signals were synchronized and recorded simultaneously.

\subsection{Skin-mounted markers measurement}

The skin-mounted marker measurement was performed by attaching active optical marker clusters and individual markers directly to the skin to track locations of anatomical landmarks which were measured by the same configuration of Visualeyez tracking system operating at $60 \mathrm{~Hz}$. The individual markers were placed directly on top of the skin of both the lateral and medial malleoli, tibial condyles, and femoral epicondyles, and of the greater trochanter, patella, and tibial tuberosity landmarks (Fig. 2) (Dumas et al., 2007). Two marker clusters with three optical markers were firmly strapped on the middle part of the shank and thigh to reconstruct the used anatomical landmarks on the tibia and femur, respectively. Reconstruction of the related landmark positions was done by performing additional measurements using a five-marker pointing device indicating landmark locations relative to the $3 \mathrm{D}$ positions of marker clusters, and the use of dedicated custom MATLAB scripts (Soderkvist and Wedin, 1993). The positions of abovementioned anatomical landmarks were also measured by individual markers to ensure that reliable continuous data were available during dynamic movements, and to minimize soft-tissue artifacts within the choice of landmarks. The placement of skin-mounted markers is demonstrated in Fig. 2. All the trajectories of attached marker clusters and individual markers accom- 


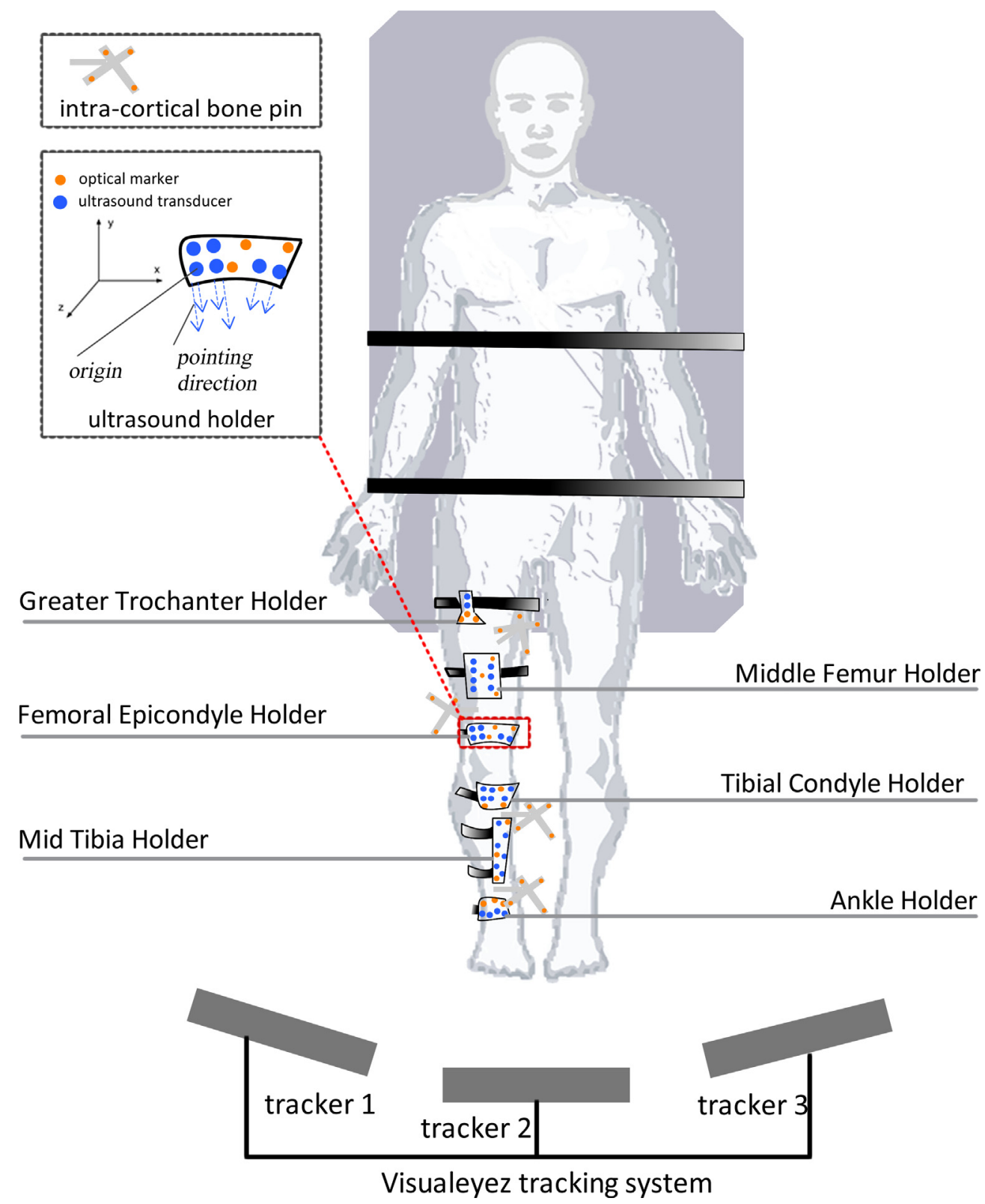

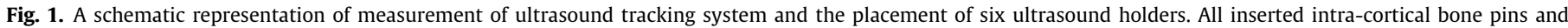
attached six ultrasound holders were tracked and recorded concurrently by Visualeyez tracking system.

panied with intra-cortical bone pins were measured and recorded simultaneously.

\subsection{Experimental trials}

Due to interferences in the placement of six ultrasound holders and skin-mounted markers altogether on the lower extremity, the cadaveric experiments needed to be performed in two independent experimental trials: (1) US-trial, i.e. ultrasound tracking system measurement with intra-cortical bone pins; (2) SKIN-trial, i.e. skin-mounted marker measurement with intra-cortical bone pins. During both trials, the same intra-cortical bone pins were used and recorded simultaneously with the other measurement technique. For each trial, the experiment consisted of a cyclic flexion and extension of the knee, performed manually. One motion cycle started from the initial pose (approximately $45^{\circ}$ of knee flexion), and proceeded to full-extension and ended back in the initial pose. The duration of one cycle was approximately $3 \mathrm{~s}$. During the experiments, we attempted to produce identical flexion-extension cycle motions that produced flexion-extension ranges of approximately from $43^{\circ}$ of flexion to $0^{\circ}$ of extension for the two different trials. At least five successive motions cycles were recorded for each trial.

\subsection{Quantification of tibiofemoral kinematics}

All measurement data for US-trial were recorded in the Diagnostic Sonar FI Toolbox (Diagnostic Sonar Ltd, Livingston, UK) on a 2.3-GHz CPU (Intel Core i7-3610QE) computer equipped with $8 \mathrm{~GB}$ of RAM. Custom written software in LabVIEW 2015 (National Instruments, Austin, Texas, US) was developed to obtain 6-DOF tibiofemoral kinematics derived from ultrasound tracking system along with corresponding ground truth kinematics obtained from intra-cortical bone pins. The detailed description of post processing can be found in (Niu et al., 2017). 


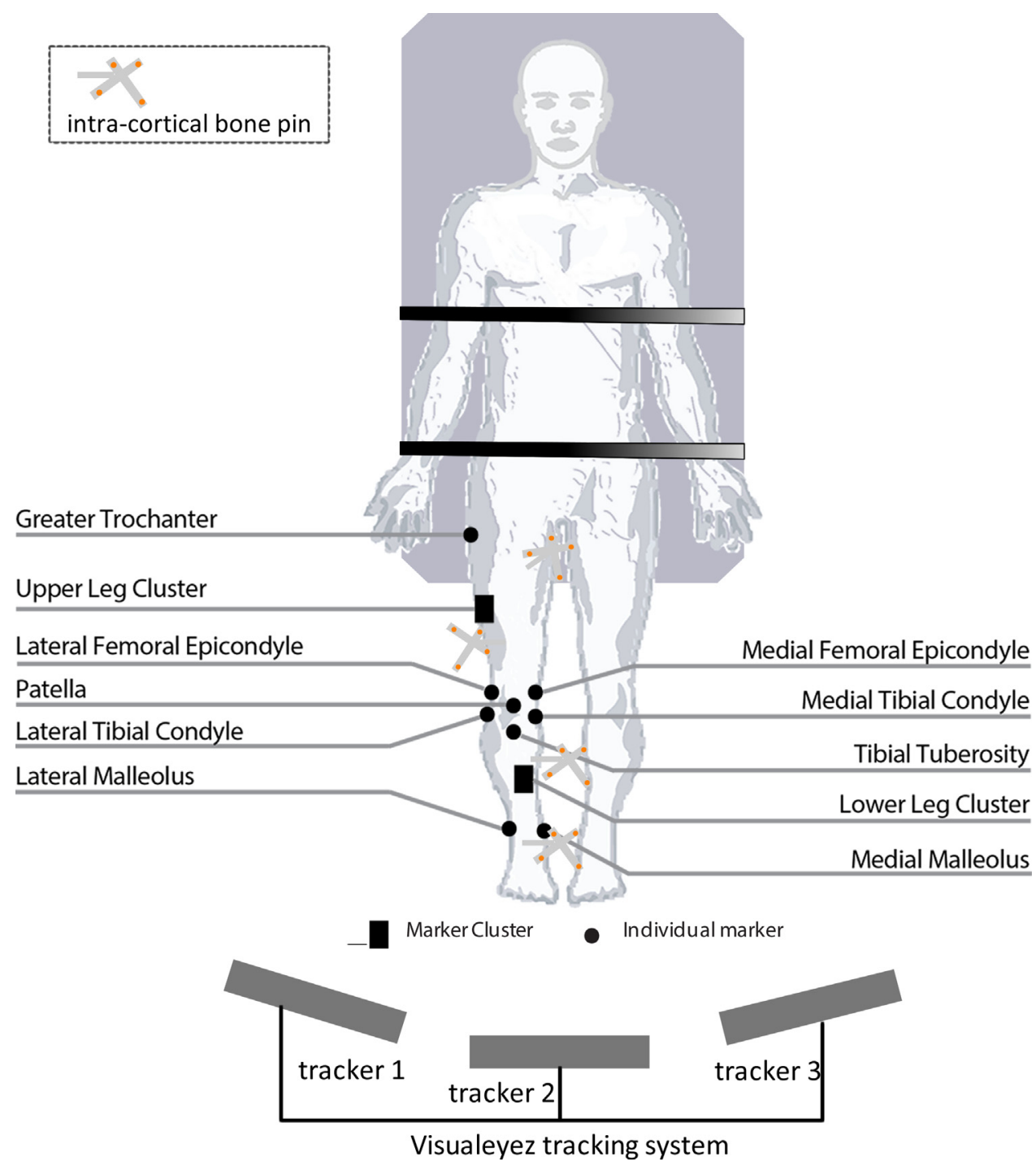

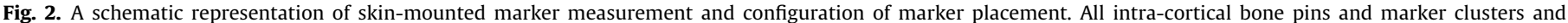
individual markers were tracked and recorded concurrently by Visualeyez tracking system.

The SKIN-trial data were firstly processed in MATLAB R2015a (MathWorks, Natick, Massachusetts, US), by applying a bidirectional second-order low-pass Butterworth filter at a cut-off frequency of $20 \mathrm{~Hz}$ onto the marker coordinates and reconstructing landmarks following marker clusters. The identical bone models and established anatomical reference frames of the femur and tibia were imported into AnyBody Modeling System (AMS, version 6.0, AnyBody Technology, Aalborg, Denmark) as used in the US-trial. Anatomical landmark locations on the bone model were defined based on bone geometry. Individual markers were used for all indicated landmarks of Fig. 2 except for the greater trochanter. The marker cluster attached to the middle thigh was used to reconstruct the pathway of the greater trochanter landmark, due to large soft-tissue artifacts within this position as a result of the cadaver setup.

Two models with different knee joint definitions were used to obtain kinematic outcomes; the hinge model and the spherical model, representing the two most widely used idealized joint definitions in kinematic analysis. Both models used the knee center of rotation as defined by the origin of the femoral anatomical reference frame (Grood and Suntay, 1983). However, the hinge model only allowed rotation in one degree of freedom, namely around the flexion-extension femoral axis, while the spherical model allowed all three rotations. Since only one kinematic DOF (flexion/extension) was allowed for the hinge model, the remaining translational and rotational kinematic components exhibited constant values. Specifically, the adduction/abduction and internal/ external rotation remained at zero, whereas the translations assumed non-zero constant values, because of the tibial anatomical reference frame being at a different location than the center of the knee joint (femoral anatomical frame). For the spherical model, the same consideration for the translations can be made as for the hinge model. Over-determinate kinematic analyses were carried out based on input spatial trajectories of processed landmarks (Andersen et al., 2009), while enforcing the relevant kinematic constraints for each of the two knee joint definitions. The corresponding ground truth kinematics (i.e. obtained from the intra-cortical bone pins) were quantified using the same routine as for the US-trial (Niu et al., 2017).

\subsection{Data analysis}

Absolute errors between the ground truth kinematics and the corresponding kinematics from the US-trial and SKIN-trial were calculated. Mean, standard deviation (SD), and root-meansquared (RMS) errors were calculated. 6-DOF tibiofemoral kine- 
matics were plotted as a function of increasing sample number for US-trial and SKIN-trial in Figs. 3 and 4, respectively. In addition, the bias of estimated kinematics using the ultrasound tracking system, the spherical model, and the hinge model with respect to their respective ground truth kinematics were computed as a function of percentage of the motion cycle (Fig. 5). The overall absolute errors for six kinematic components were illustrated in box whisker plots to intuitively display the variety of estimated kinematic errors for the different methods (Fig. 6).

\section{Results}

The mean rotational errors ranged from $3.23^{\circ}$ to $6.25^{\circ}$ and from $2.17^{\circ}$ to $6.12^{\circ}$ for the spherical model and hinge model, respectively. The mean translational errors ranged from 4.65 to 5.82 $\mathrm{mm}$ and from 4.56 to $6.39 \mathrm{~mm}$ for the spherical model and hinge model, respectively. For the ultrasound tracking system, the mean rotational errors $\left(0.85^{\circ}-2.65^{\circ}\right)$ and mean translational errors $(2.00-4.35 \mathrm{~mm})$ were lower than those errors of the two types of

\section{US-trial}
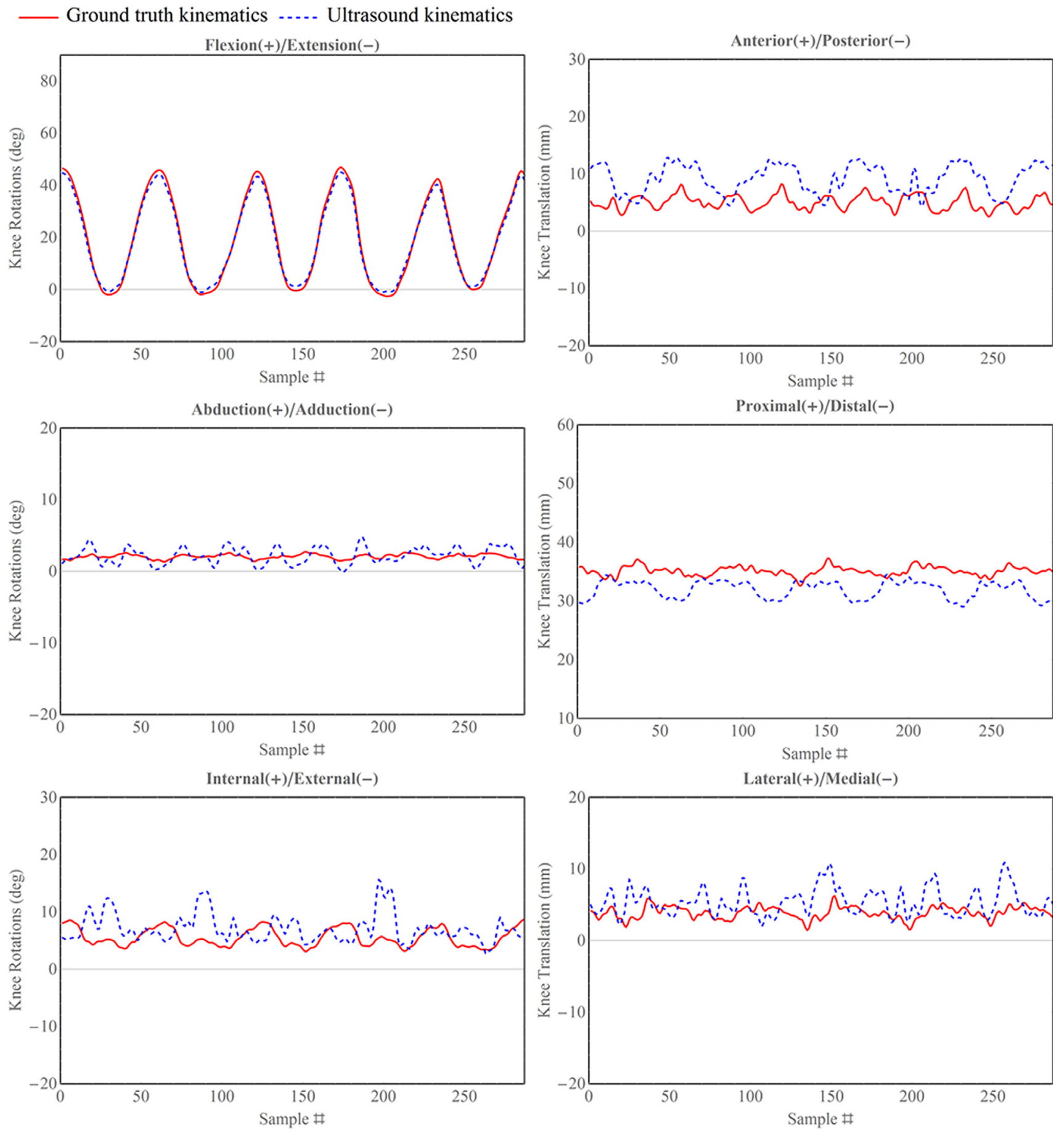

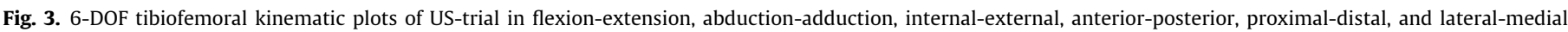

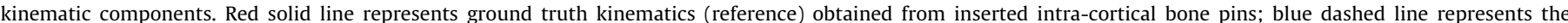

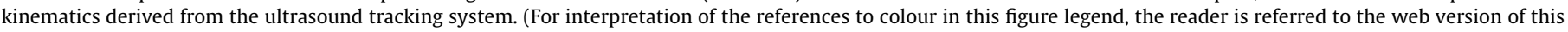
article.) 


\section{SKIN-trial}

\section{.... Hinge joint kinematics}
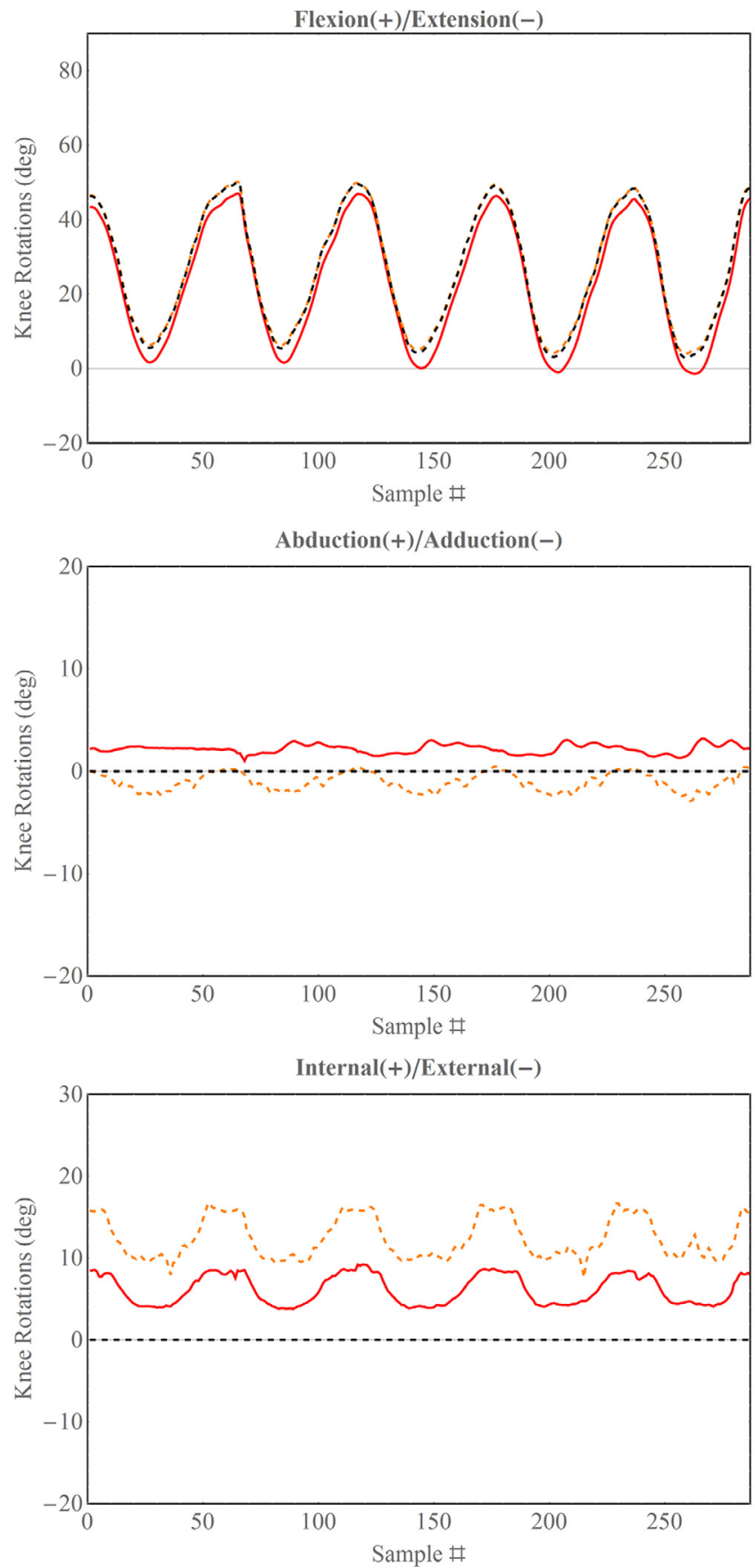

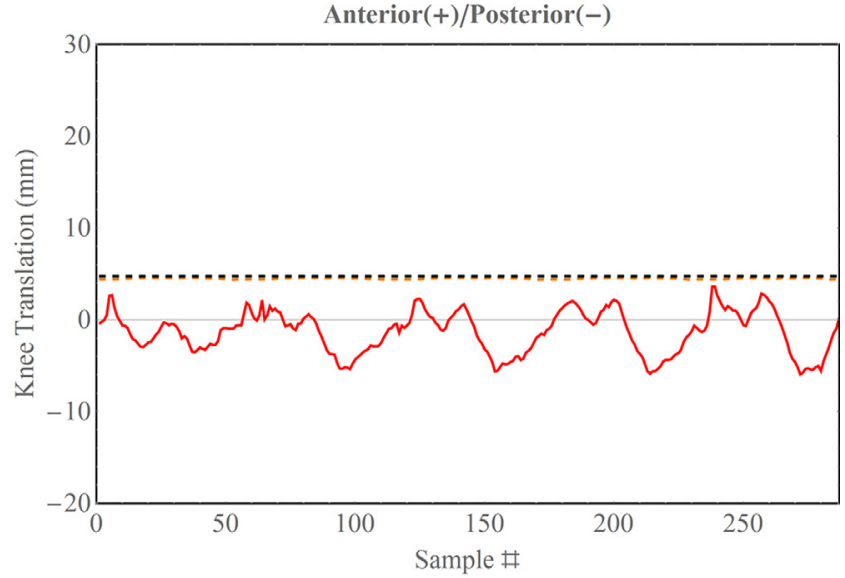

Proximal(+)/Distal(-)

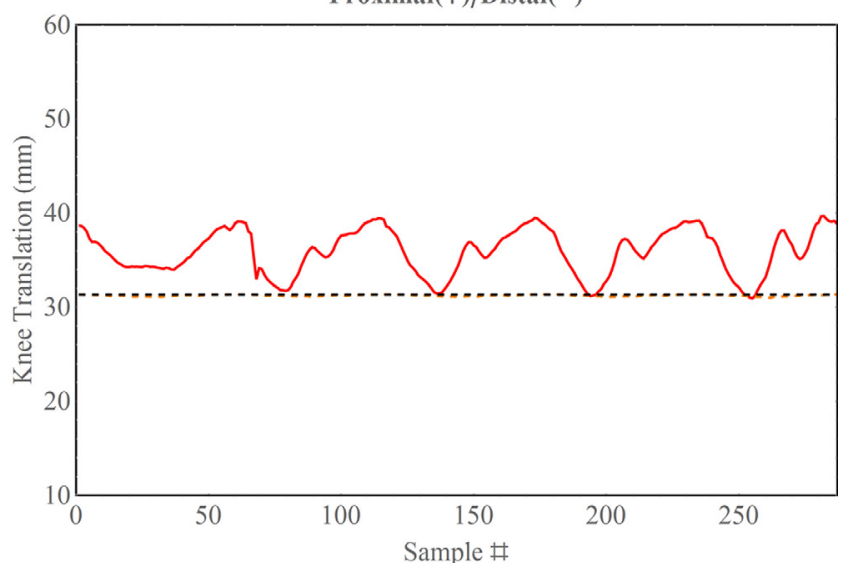

Lateral $(+) / \operatorname{Medial}(-)$

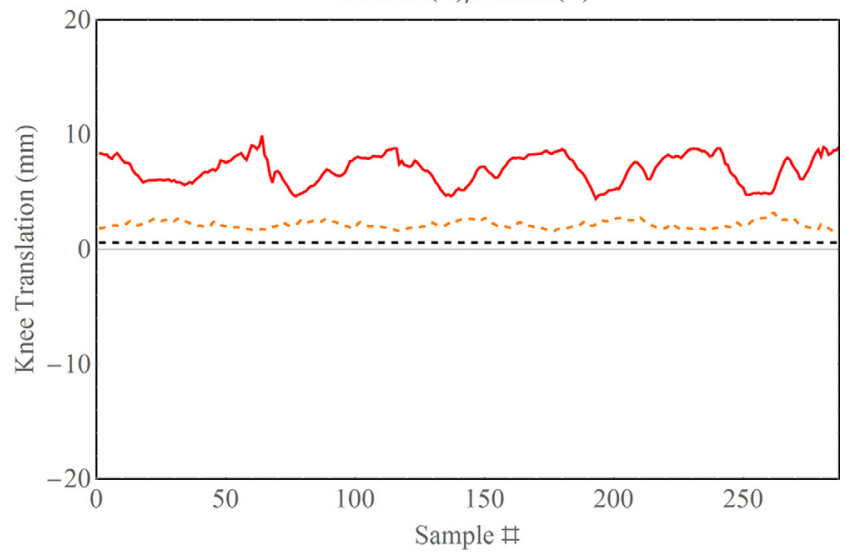

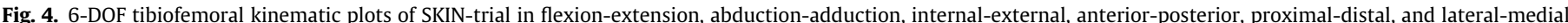

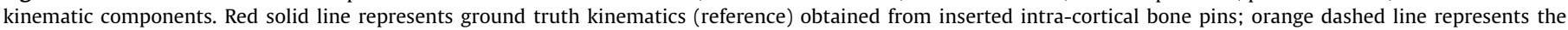

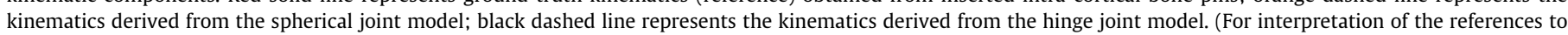
colour in this figure legend, the reader is referred to the web version of this article.)

kinematic measurements (obtained from the spherical and hinge models, Table 1). Similarly lower errors were found in RMS errors for the ultrasound tracking system $\left(1.00^{\circ}-3.44^{\circ}\right.$ and $2.52-4.88$ $\mathrm{mm})$ compared with the spherical model $\left(3.38^{\circ}-6.32^{\circ}\right.$ and 5.04 $6.26 \mathrm{~mm})$ and the hinge model $\left(2.22^{\circ}-6.38^{\circ}\right.$ and $\left.5.14-6.52 \mathrm{~mm}\right)$ (Table 2).

\section{Discussion}

In this study, we performed cadaveric experiments in order to compare the measurement of our novel ultrasound tracking system with skin-mounted marker measurement for assessing the accuracy in estimated tibiofemoral kinematics in a highly con- 
ultrasound tracking system
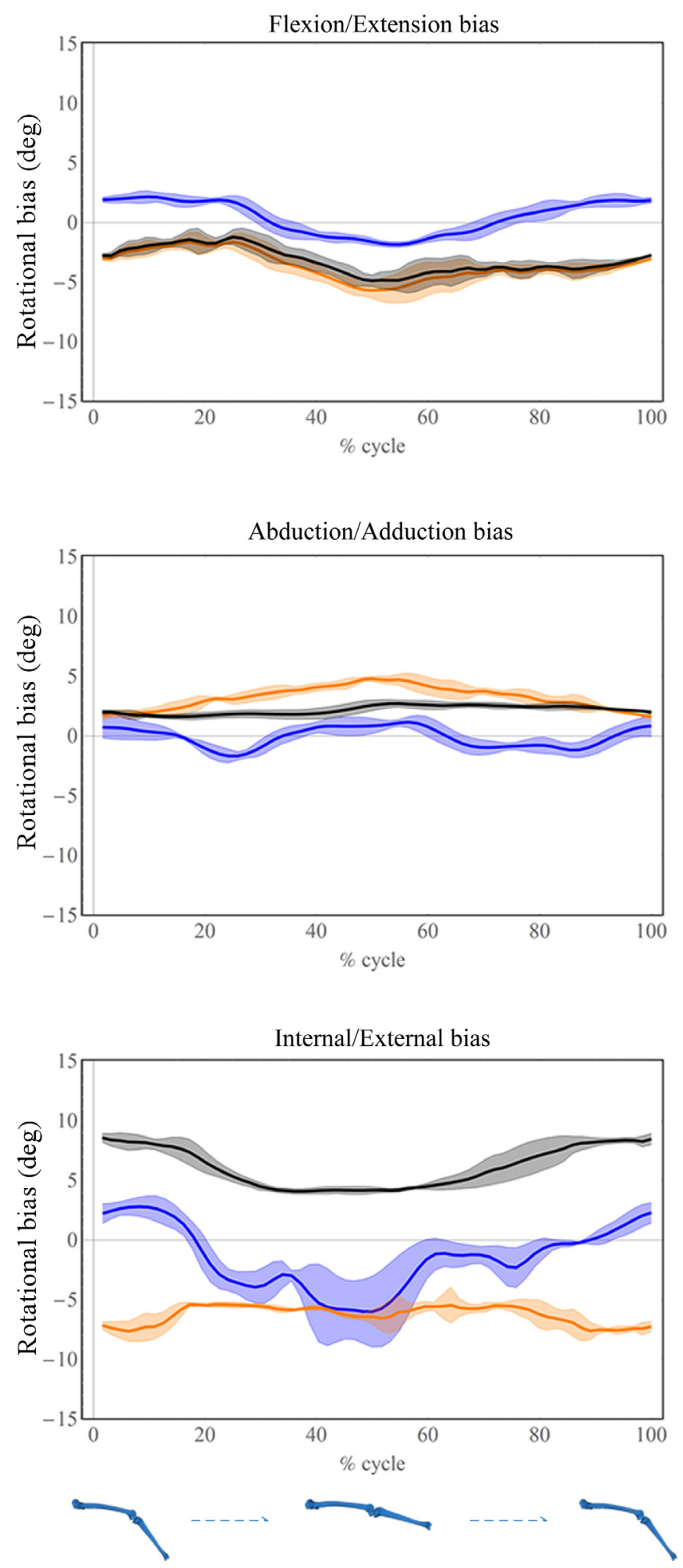
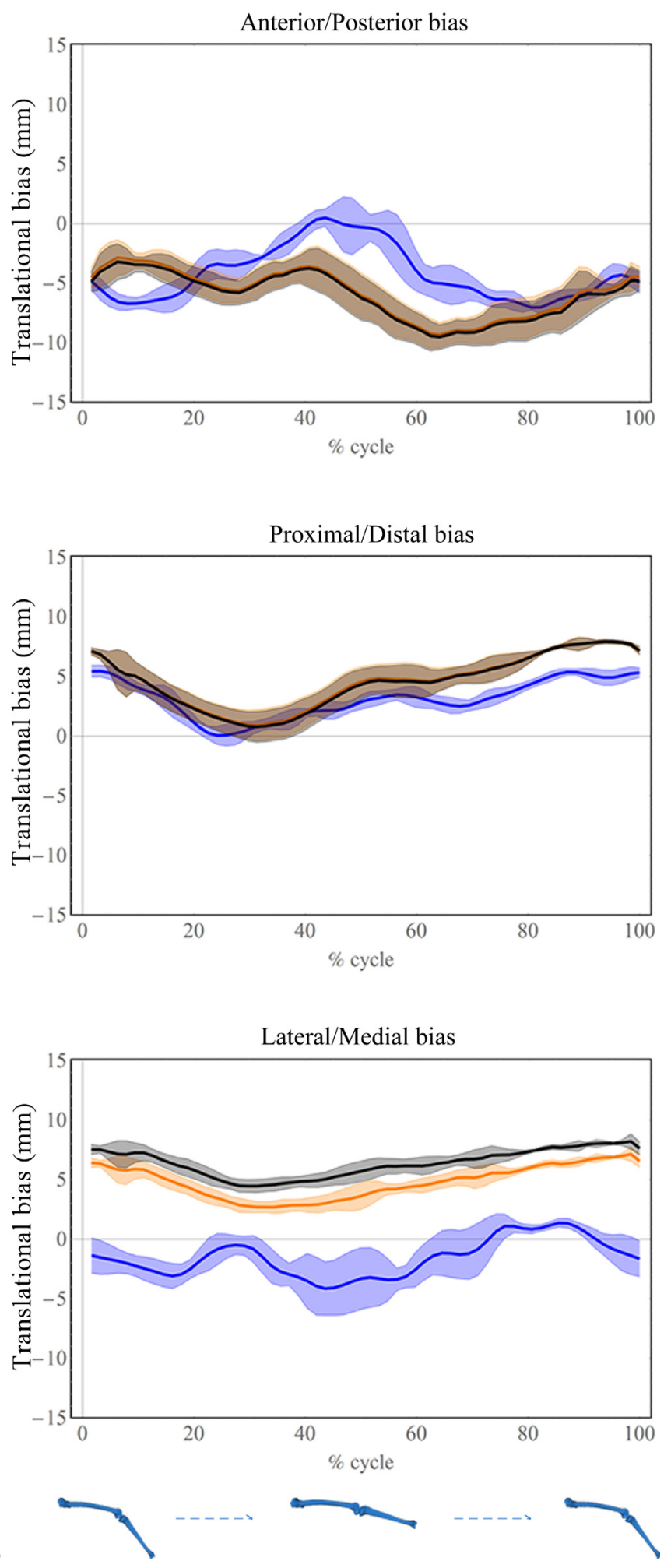

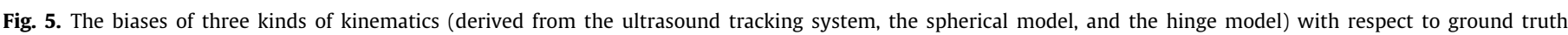

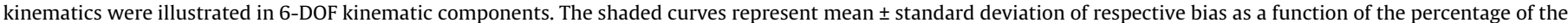

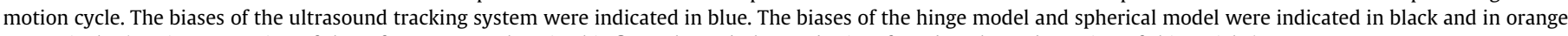
respectively. (For interpretation of the references to colour in this figure legend, the reader is referred to the web version of this article.)

trolled experimental scenario. As the results have shown, the ultrasound tracking system could achieve relatively high accuracies in flexion/extension (1.54 ${ }^{\circ}$ RMS error) and abduction/adduction
(1.00 ${ }^{\circ}$ RMS error), which is close to the accuracy of the mobile fluoroscopic system with $0.77^{\circ}$ RMS error (Guan et al., 2016a). In addition, the ultrasound tracking system showed the potential to 

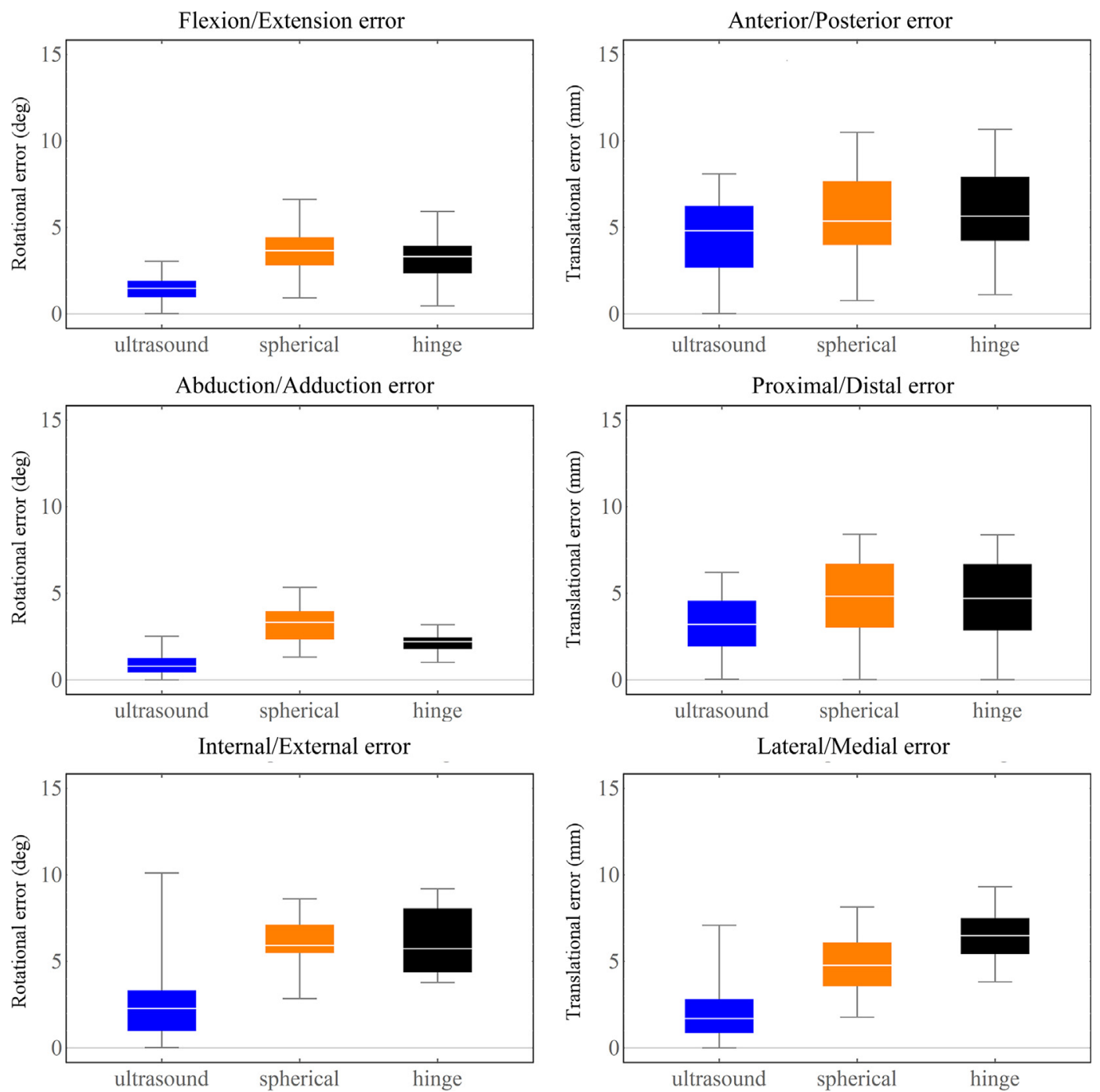

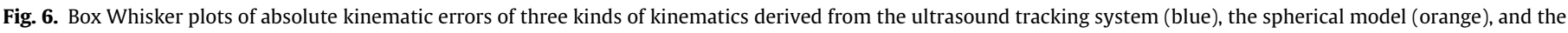

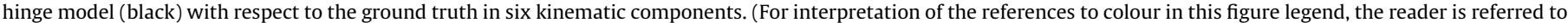
the web version of this article.)

Table 1

Mean \pm standard deviation (SD) of absolute errors describing the errors associated with three types of kinematic measurements (the ultrasound tracking system, the spherical joint model, and the hinge joint model).

\begin{tabular}{llll}
\hline Mean \pm SD & Ultrasound tracking system & Spherical joint & Hinge joint \\
\hline Flex/Ext $\left(^{\circ}\right)$ & $1.39 \pm 0.66$ & $3.62 \pm 1.35$ & $3.20 \pm 1.24$ \\
Abd/Add $\left(^{\circ}\right)$ & $0.85 \pm 0.53$ & $3.23 \pm 1.01$ & $2.17 \pm 0.44$ \\
Int/Ext $\left(^{\circ}\right)$ & $2.65 \pm 2.20$ & $6.25 \pm 0.98$ & $6.12 \pm 1.79$ \\
Ant/Post $(\mathrm{mm})$ & $4.35 \pm 2.21$ & $5.82 \pm 2.31$ & $6.06 \pm 2.29$ \\
Prox/Dist $(\mathrm{mm})$ & $3.14 \pm 1.61$ & $4.65 \pm 2.34$ & $4.56 \pm 2.38$ \\
Lat/Med $(\mathrm{mm})$ & $2.00 \pm 1.53$ & $4.82 \pm 1.48$ & $6.39 \pm 1.25$ \\
\hline
\end{tabular}

Table 2

Root-mean-square (RMS) error describing the errors associated with three types of kinematic measurements (ultrasound tracking system, spherical joint model, and hinge joint model).

\begin{tabular}{llll}
\hline RMS error & Ultrasound tracking system & Spherical joint & Hinge joint \\
\hline Flex/Ext $\left({ }^{\circ}\right)$ & 1.54 & 3.86 & 3.43 \\
Abd/Add $\left(^{\circ}\right)$ & 1.00 & 3.38 & 2.22 \\
Int/Ext $\left(^{\circ}\right)$ & 3.44 & 6.32 & 6.38 \\
Ant/Post $(\mathrm{mm})$ & 4.88 & 6.26 & 6.47 \\
Prox/Dist $(\mathrm{mm})$ & 3.53 & 5.20 & 5.14 \\
Lat/Med $(\mathrm{mm})$ & 2.52 & 5.04 & 6.52 \\
\hline
\end{tabular}

measure all 6-DOF kinematics with comparable accuracy by effectively reducing the propagation of STA to kinematic outcomes. In this study, the lower accuracy in internal/external rotation $\left(3.44^{\circ}\right.$ RMS error) indicated the instability of the ultrasound tracking system when the leg was at full-extension pose. We believed this mainly originates from the lack of skin contact for several ultrasound holders caused by the deformations of surrounding soft tissue when stretching. Further research will focus on the improvement of the design of the ultrasound holders, especially regarding to skin contact.

Different to conventional motion capture systems using skinmounted markers, we introduced an ultrasound technique into kinematic measurement, thus taking advantage of the capability of ultrasound transducer to measure underlying skeletal structure through soft tissues, which is an extension of the navigated Amode ultrasound probes utilized in computer assisted orthopedic surgery (Amstutz et al., 2003; Chang et al., 2009; Fieten et al., 2009; Heger et al., 2002). In this study, an A-mode ultrasound transducer (single element ultrasound transducer) could be deemed as a virtually extended measurement line from the skin to the bone surface. Some researchers have proposed a concept utilizing A-mode ultrasound for a sweep scan on the femur to measure $3 \mathrm{D}$ motion through the image registration method (Masum 
et al., 2014b). The reported accuracy was in the order of submillimeter and sub-degree. However, that study was performed when a sawbone model of the femur was immersed in a water tank. The related range of motion was relative small, only $\pm 4 \mathrm{~mm}$ and $\pm 3^{\circ}$. On the other hand, the sweep scan of A-mode to generate a 2D image was similar to B-mode ultrasound acquisition, but typically has a lower sample rate. In principle that concept is different than our proposed A-mode ultrasound tracking system, since we employed multiple A-mode transducers at different anatomical locations for discrete point acquisition. Other researchers combined B-mode ultrasound transducer with a motion capture system, which could obtain one or multiple curves representing bone surfaces (Jia et al., 2017; Masum et al., 2014a). The potential of being less affected by STA has been validated in an in-vivo experiment by merely measuring the depth of bone surfaces on the greater trochanter with up to $16 \mathrm{~mm}$ measurement difference to skin marker measurement (Jia et al., 2017). However, to our knowledge, dynamic tibiofemoral kinematic measurements have not been performed or validated in previous studies.

The framework for processing measured datasets of skinmounted markers has been developed by many researchers, containing different kinds of MKO models besides the hinge and spherical models which were used for comparison in this study, e.g. parallel (Duprey et al., 2010), coupling (Bonnechère et al., 2015), and elastic (Richard et al., 2016) models. The placement and configuration of skin-mounted markers have also been investigated to assess the STA measurement error (Garling et al., 2007). Although those findings showed a certain extent of mitigation of measurement errors related to STA, the validity of representing actual position and orientation of bone segments and related joint kinematics have not been improved (Andersen et al., 2010; Richard et al., 2017). This difficulty stems from the principle that processing skin-mounted markers cannot provide straightforward measurement of the underlying bone (Leardini et al., 2005). Current models and mathematical optimizations are unable to tackle this difficulty completely. Only invasive or fluoroscopic methods are currently capable of assessing joint kinematics with desirable level of accuracy of ( $2^{\circ}$ and $2 \mathrm{~mm}$ ) (Richard et al., 2017). Based on the current study, the ultrasound tracking system showed the potential of being considered as an alternative to measure joint kinematics in the near future. It provides a new approach of being less affected by STA by actually measuring bone surfaces. Thus, this approach also has the potential to mitigate the effect of STA and to reduce the propagation of STA to final kinematics. In addition, no matter how the ultrasound holder shifts with respect to the underlying bone (i.e. either ultrasound holder to skin or skin to bone movements), as long as the underlying bone segment remains within the "field of view" of ultrasound transducer, valid bony points can be captured and the registration algorithm could find an appropriate transformation to match the bone model to the point cloud of bony points and quantify 6-DOF tibiofemoral kinematics. When using skin-mounted markers, this type of shifting would contaminate the quality of skin-mounted marker dataset.

This work has several limitations. First, only one cadaveric specimen was used for the experiments. Ideally, a cohort of living subjects covering different sizes and BMIs and using advanced biplane fluoroscopic systems as the ground truth measurement could provide more valuable information regarding to STA and its effects on kinematics, which is related to an in-vivo validation study of the ultrasound tracking system for future work. Second, the ultrasound tracking system and skin-mounted marker measurement were used consecutively instead of simultaneously. However, using two independent measurement trials accompanied with the same intra-cortical bone pins serving as ground truth makes the quantitative comparison feasible and justifiable in this study. Third, only hinge and spherical models were used for comparison, which have their own limited DOF in final kinematic outcomes. However, these two models are the most widely used representations of the knee joint in motion analysis. Fourth, knee motion of cadaveric leg was manipulated manually and the posture of body differed from the normal posture, e.g. walking. Subsequently, the muscle and soft tissue were passively stretched and compressed, which also differed from those during gait. However, to reproduce the locomotion of normal gait is quite challenging in a cadaveric setting. Fifthly, the sample rate of the whole ultrasound tracking system was $20 \mathrm{~Hz}$ in this experiment, which may impede its application on dynamic motions. However, the sample rate can be improved by updating hardware and optimizing the data acquisition procedure. In addition, the cable of ultrasound transducers may also influence or hinder normal motions and increase complexity of this system when employed in a clinical application. However, this cable issue can be mitigated by carefully redesigning the ultrasound holders or the advances of new data transmission technique, e.g. wireless communication. Further studies will move towards the assessment and validation of the ultrasound tracking system on the living subjects performing daily activities. To realize this goal, some improvements will be completed first, such as the redesign of the ultrasound holders for better skin contact and the employment of an advanced registration algorithm. In this context, MRI scanning will be used to replace CT scanning for in-vivo application because of the radiation issue. With the high potential to implement further improvements associated with the ultrasound tracking system, we expect that a future ultrasound tracking system could estimate 6-DOF tibiofemoral kinematics with an accuracy within the range of fluoroscopy measurements (Guan et al., 2016a, 2016b) and provide a valid representation of bone segments in the knee joint, which will aid in gait analysis and disclosure of some pathological features.

\section{Conclusion}

We proposed a quantitative comparison of tibiofemoral kinematics estimated using an ultrasound based tracking system versus traditional skin-mounted markers system with hinge and spherical knee joint models. The ultrasound tracking method resulted in lower kinematic errors, in the experimental conditions investigated, and could represent a viable alternative to traditional system, which could improve the measurement accuracy of bone and joint kinematics. This new ultrasound based kinematic measurement technique thus provides a useful approach to study joint motion for musculoskeletal modeling and biomechanical research communities.

\section{Acknowledgements}

The research leading to these results has received funding from the European Research Council under the European Union's Seventh Framework Programme (FP/2007-2013)/ERC Grant Agreement n. 323091 awarded to N. Verdonschot. The authors thank for the generous helps of Léon Driessen and Richard van Swam in the cadaveric preparation from Orthopedic Research Lab, Radboud University Medical Center, Netherlands. The authors also thank for the technical help of Mats Boeve in the design of ultrasound holders from University of Twente, Netherlands.

\section{Conflict of interest statement}

The authors have no conflict of interests to report related to this work. 


\section{References}

Akbarshahi, M., Schache, A.G., Fernandez, J.W., Baker, R., Banks, S., Pandy, M.G. 2010. Non-invasive assessment of soft-tissue artifact and its effect on knee joint kinematics during functional activity. J. Biomech. 43, 1292-1301.

Amstutz, C., Caversaccio, M., Kowal, J., Bachler, R., Nolte, L.P., Hausler, R., Styner, M., 2003. A-mode ultrasound-based registration in computer-aided surgery of the skull. Archives of otolaryngology-head \& neck surgery 129, $1310-1316$.

Andersen, M.S., Benoit, D.L., Damsgaard, M., Ramsey, D.K., Rasmussen, J., 2010. Do kinematic models reduce the effects of soft tissue artefacts in skin markerbased motion analysis? An in vivo study of knee kinematics. J. Biomech. 43, $268-273$.

Andersen, M.S., Damsgaard, M., Rasmussen, J., 2009. Kinematic analysis of overdeterminate biomechanical systems. Comput. Methods Biomech Biomed. Eng. $12,371-384$.

Benoit, D.L., Ramsey, D.K., Lamontagne, M., Xu, L., Wretenberg, P., Renström, P., 2006. Effect of skin movement artifact on knee kinematics during gait and cutting motions measured in vivo. Gait Posture 24, 152-164.

Besl, P.J., McKay, H.D., 1992. A method for registration of 3-D shapes. IEEE Trans. Pattern Anal. Mach. Intell. 14, 239-256.

Bonnechère, B., Sholukha, V., Salvia, P., Rooze, M., Van Sint Jan, S., 2015 Physiologically corrected coupled motion during gait analysis using a modelbased approach. Gait Posture 41, 319-322.

Cappozzo, A., Cappello, A., Croce, U.D., Pensalfini, F., 1997. Surface-marker cluster design criteria for 3-D bone movement reconstruction. IEEE Trans. Biomed. Eng. 44, 1165-1174.

Cappozzo, A., Catani, F., Croce, U.D., Leardini, A., 1995. Position and orientation in space of bones during movement: anatomical frame definition and determination. Clin. Biomech. (Bristol, Avon) 10, 171-178.

Cappozzo, A., Catani, F., Leardini, A., Benedetti, M.G., Della Croce, U., 1996. Position and orientation in space of bones during movement: experimental artefacts. Clin. Biomech. 11, 90-100.

Cereatti, A., Bonci, T., Akbarshahi, M., Aminian, K., Barre, A., Begon, M., Benoit, D.L., Charbonnier, C., Dal Maso, F., Fantozzi, S., Lin, C.C., Lu, T.W., Pandy, M.G., Stagni, R., van den Bogert, A.J., Camomilla, V., 2017. Standardization proposal of soft tissue artefact description for data sharing in human motion measurements. J. Biomech.

Cereatti, A., Della Croce, U., Cappozzo, A., 2006. Reconstruction of skeletal movement using skin markers: comparative assessment of bone pose estimators. J. NeuroEng. Rehabil. 3, 7.

Chang, T.C., Mozes, A., Arata, L., Zhao, W., 2009. A-Mode Ultrasound Bone Registration for Computer-Assisted Knee Surgery: Calibration and Robustness Test. In: McGoron, A., Li, C.-.Z., Lin, W.-.C. (Eds.), 25th Southern Biomedica Engineering Conference 2009, 15-17 May 2009, Miami, Florida, USA. Springer, Berlin Heidelberg, pp. 97-100.

Charlton, I.W., Tate, P., Smyth, P., Roren, L., 2004. Repeatability of an optimised lower body model. Gait Posture 20, 213-221.

Clement, J., Dumas, R., Hagemeister, N., de Guise, J.A., 2017. Can generic knee joint models improve the measurement of osteoarthritic knee kinematics during squatting activity? Comput. Methods Biomech. Biomed. Eng. 20, 94-103.

Dumas, R., Cheze, L., Verriest, J.P., 2007. Adjustments to McConville et al. and Young et al. body segment inertial parameters. J. Biomech. 40, 543-553.

Dumas, R., Jacquelin, E., 2017. Stiffness of a wobbling mass models analysed by a smooth orthogonal decomposition of the skin movement relative to the underlying bone. J. Biomech.

Duprey, S., Cheze, L., Dumas, R., 2010. Influence of joint constraints on lower limb kinematics estimation from skin markers using global optimization. J. Biomech. 43, 2858-2862.
Fieten, L., Schmieder, K., Engelhardt, M., Pasalic, L., Radermacher, K., Heger, S., 2009. Fast and accurate registration of cranial CT images with A-mode ultrasound. Int. J. Comput. Assist. Radiol. Surg. 4, 225-237.

Garling, E.H., Kaptein, B.L., Mertens, B., Barendregt, W., Veeger, H.E., Nelissen, R.G., Valstar, E.R., 2007. Soft-tissue artefact assessment during step-up using fluoroscopy and skin-mounted markers. J. Biomech. 40 (Suppl 1), S18-24.

Grood, E.S., Suntay, W.J., 1983. A joint coordinate system for the clinical description of three-dimensional motions: application to the knee. J. Biomech. Eng. 105 136-144.

Guan, S., Gray, H.A., Keynejad, F., Pandy, M.G., 2016a. Mobile biplane X-ray imaging system for measuring 3D dynamic joint motion during overground gait. IEEE Trans. Med. Imag. 35, 326-336.

Guan, S., Gray, H.A., Schache, A.G., Feller, J., de Steiger, R., Pandy, M.G., 2016b. In vivo six-degree-of-freedom knee-joint kinematics in overground and treadmill walking following total knee arthroplasty. J. Orthopaedic Res., n/a-n/a.

Heger, S., Portheine, F., Ohnsorge, J., Radermacher, K., 2002. Visual user guidance for registration based on A-mode ultrasound. Biomedizinische Technik. Biomed. Eng. 47 (Suppl 1 Pt 1), 61-64.

Jia, R., Monk, P., Murray, D., Noble, J.A., Mellon, S., 2017. CAT \& MAUS: A novel system for true dynamic motion measurement of underlying bony structures with compensation for soft tissue movement. J. Biomech.

Lafortune, M.A., Cavanagh, P.R., Sommer, H.J., Kalenak, A., 1992. Three-dimensional kinematics of the human knee during walking. J. Biomech. 25, 347-357.

Leardini, A., Chiari, L., Croce, U.D., Cappozzo, A., 2005. Human movement analysis using stereophotogrammetry: part 3. Soft tissue artifact assessment and compensation. Gait Posture 21, 212-225.

Lu, T.W., O'Connor, J.J., 1999. Bone position estimation from skin marker coordinates using global optimisation with joint constraints. J. Biomech. 32, 129134.

Ma, B., Ellis, R.E., 2003. Robust registration for computer-integrated orthopedic surgery: laboratory validation and clinical experience. Med. Image Anal. 7, 237250.

Masum, M.A., Pickering M., Lambert, A., Scarvell, J., Smith, P., 2014a. Accuracy assessment of Tri-plane B-mode ultrasound for non-invasive 3D kinematic analysis of knee joints. Biomed. Eng. Online 13, 122.

Masum, M.A., Pickering, M.R., Lambert, A.J., Scarvell, J.N., Smith, P.N., 2014b. Precision analysis of single-element ultrasound sensor for Kinematic analysis of knee joints. Electron. Lett. 50, 1047-1048.

Miranda, D.L., Rainbow, M.J., Leventhal, E.L., Crisco, J.J., Fleming, B.C., 2010. Automatic determination of anatomical coordinate systems for threedimensional bone models of the isolated human knee. J. Biomech. 43, 16231626.

Niu, K., Sluiter, V., Sprengers, A., Homminga, J., Verdonschot, N., 2017. A novel tibiafemoral kinematics measurement system based on multi-channel A-mode ultrasound system. In: CAOS 2017. 17th Annual Meeting of the International Society for Computer Assisted Orthopaedic Surgery. Aachen, Germany.

Ojeda, J., Martinez-Reina, J., Mayo, J., 2014. A method to evaluate human skeletal models using marker residuals and global optimization. Mech. Mach. Theory 73, $259-272$.

Reinbolt, J.A., Schutte, J.F., Fregly, B.J., Koh, B.I., Haftka, R.T., George, A.D., Mitchell, K. H., 2005. Determination of patient-specific multi-joint kinematic models through two-level optimization. J. Biomech. 38, 621-626.

Richard, V., Cappozzo, A., Dumas, R., 2017. Comparative assessment of knee joint models used in multi-body kinematics optimisation for soft tissue artefact compensation. J. Biomech.

Richard, V., Lamberto, G., Lu, T.W., Cappozzo, A., Dumas, R., 2016. Knee kinematics estimation using multi-body optimisation embedding a knee joint stiffness matrix: a feasibility study. PloS one 11, e0157010.

Soderkvist, I., Wedin, P.A., 1993. Determining the movements of the skeleton using well-configured markers. J. Biomech. 26, 1473-1477. 